SCIENTIFIC LETTER

\title{
A novel method of assessing Starr-Edwards aortic valve stenosis
}

\author{
R J Edwards, M Al-Bustami, C D J Ilsley
}

Heart 2004;90:1479-1480. doi: 10.1136/hrt.2003.020784

$C^{2}$ atheter assessment of Starr-Edwards prosthetic aortic valve stenosis requires simultaneous recording of left ventricular (LV) and aortic pressures. Passage of conventional Dacron catheters across mechanical valves can damage the mechanism and induce valvar incompetence. The alternative Bockenbrough procedure requires atrial septal puncture with potential lethal complications. ${ }^{1}$ The availability of a low profile, floppy tipped, 0.014 inch guidewire mounted pressure sensor (PressureWire 4, RADI Medical Systems, Uppsala, Sweden) provides a novel means of crossing this type of valve. The use of fine bore pressure wires may be less likely to disturb the ball cage mechanism than larger fluid filled catheters, and may provide a safe and reproducible method of assessing Starr-Edwards aortic valve stenosis. However, the use of the pressure wire in assessing bileaflet or tilting disc prosthetic valves may be more problematic.

\section{CASE HISTORY}

A 54 year old man presented to clinic with a six month history of worsening shortness of breath. He had had a size 10 A Starr-Edwards aortic valve inserted in 1990 and had persistent atrial fibrillation. He had no other significant comorbidity. Clinical examination revealed systolic murmurs at the apex and aortic areas. An echocardiogram demonstrated a left ventricle with end diastolic and end systolic diameters at the upper limit of normal with overall good function, moderate mitral regurgitation, and a variable peak aortic gradient estimated from continuous wave Doppler at 40$80 \mathrm{~mm} \mathrm{Hg}$.

We proceeded to investigate with cardiac catheterisation. Right heart pressures were recorded conventionally with a 7 French multipurpose catheter. Left heart catheterisation was performed from the right femoral artery with a 6 French sheath.

The pressure wire was calibrated and advanced through a 6 French JR4 guiding catheter. Initial attempts were unsuccessful at crossing into the left ventricle; the tip of the wire did not feel sufficiently robust to bypass the ball. Further support was obtained from an over the wire angioplasty balloon, which enabled the floppy tip and pressure transducer to prolapse through the cage and sense the pressure in the left ventricle. Simultaneous aortic pressure was recorded from the guide catheter and both pressure recordings are shown in fig 1. Cardiac pressures were as follows: pulmonary artery mean $43 \mathrm{~mm} \mathrm{Hg}$; pulmonary capillary wedge pressure (atrial wave/ventricular wave/mean 29/38/26 mm Hg; LV (systolic/end diastolic) 119/ $35 \mathrm{~mm} \mathrm{Hg}$; aortic (systolic/diastolic) 108/72 mm Hg. These findings demonstrated no evidence of aortic stenosis, which was in contrast to the estimated Doppler gradient. The elevated pulmonary wedge pressure and large $\mathrm{V}$ wave indicated that the mitral regurgitation was the most significant lesion.
Figure 1 Simultaneous pressure recordings in the aorta and left ventricle. The lower most trace is recorded from the PressureWire. The uppermost trace is recorded though the fluid filled guide catheter in the aorta. In the second wave form the pressure wire has prolapsed from the cage into the left ventricle. There is a trivial difference in peak systolic LV pressure and systolic aortic pressure indicating no significant restriction of flow across the valve.

\section{DISCUSSION}

It is well documented that the pressure drop across prosthetic valves measured by Doppler echo may be significantly greater than that recorded directly with conventional manometric techniques. ${ }^{2-5}$ This is because the continuous wave Doppler measures the initial loss of hydrostatic pressure directly at the valve orifice. Previous studies have shown that there is then partial recovery of the pressure in the proximal aorta as the post-stenotic jet expands. ${ }^{45}$ This is the pressure recorded by the fluid filled catheter and explains why the transprosthetic gradient measured through the valve orifice is higher than that measured downstream in the aorta. $^{6}$

The pressure wire was developed for the assessment of fractional flow reserve in coronary arteries. It is the most frequently used device that uses high fidelity sensor tipped wire technology. To our knowledge it has never been used to assess gradients across mechanical valves. However, this case report demonstrates a novel method of measuring LV pressure in a patient with a Starr-Edwards mechanical heart valve. It must be emphasised that this method should never be used in tilting disc or bileaflet prostheses because of the risk of jamming the occluder in a closed position. 
This case report describes a novel technique of simultaneously measuring LV pressure and aortic pressure in a patient with suspected stenosis in a Starr-Edwards aortic valve. Echocardiography can overestimate the gradient across mechanical valves, and direct measurement of aortic and LV pressure in this patient was desirable. However, passage of conventional Dacron catheters across mechanical valves can damage the mechanism and induce valvar incompetence; the alternative Bockenbrough procedure requires atrial septal puncture with potential lethal complications. The availability of a low profile, floppy tipped, 0.014 inch guidewire mounted pressure sensor (PressureWire 4) provides a novel means of crossing this type of valve. In this case we used a fine bore pressure wire which may be less likely to disturb the ball cage mechanism than larger fluid filled catheters, and provided a novel method of assessing Starr-Edwards aortic valve stenosis.

\section{Authors' affiliations}

R J Edwards, M Al-Bustami, C D J Ilsley, Harefield Hospital, Middlesex, UK
Correspondence to: Dr Richard J Edwards, Harefield Hospital, Hill End Road, Harefield, Middlesex UB9 6JH, UK; r.edwards@rbh.nthames. nhs.uk

\section{Accepted 5 March 2004}

\section{REFERENCES}

1 Bockenbrough EC, Braunwald E, Ross J, et al. Transseptal left heart catheterization. A review of 450 studies and description of an improved technique. Circulation 1962;25:15-21.

2 Chakraborty B, Quek S, Pin DZ, et al. Doppler echocardiographic assessment of normally functioning Starr-Edwards, carbomedics and Carpentier-Edwards valves in aortic position. Angiology 1996:47:481-9.

3 Rashtian MY, Stevenson DM, Allen DT, et al. Flow characteristics of four commonly used mechanical heart valves. Am J Cardiol 1986;58:743-52.

4 Baumgartner $\mathbf{H}$, Khan S, DeRobertis $M$, et al. Effect of prosthetic aortic valve design on the Doppler-catheter gradient correlation: an in vitro study of normal St. Jude, Medtronic-Hall, Starr-Edwards and Hancock valves. J Am Coll Cardiol 1992;19:324-32.

5 Rothbart RM, Smucker ML, Gibson RS. Overestimation by Doppler echocardiography of pressure gradients across Starr-Edwards prosthetic valves in the aortic position. Am J Cardiol 1988;61:475-6.

6 Marcus RH, Heinrich RS, Bednarz J, et al. Assessment of small-diameter aortic mechanical prostheses: physiological relevance of the Doppler gradient, utility of flow augmentation, and limitations of orifice area estimation. Circulation 1998;98:866-72

\section{IMAGES IN CARDIOLOGY}

\section{Multislice cardiac computed tomography of symmetry bypass connector}

doi: $10.1136 /$ hrt.2004.034355

A 66 year old man underwent coronary artery bypass for severe three vessel coronary disease on 18 September 2002. Conduits included a left internal mammary artery (LIMA) to the left internal descending artery (LAD), free radial artery bypass to his first obtuse marginal (OM), and a saphenous vein graft ( $S V G$ ) to the posterior descending artery (PDA). The SVG anastomosis was created on the ascending aorta using a Symmetry bypass connector (St Jude Medical, St Paul, Minnesota, USA).

As part of an ongoing research protocol to evaluate the feasibility of bypass conduit imaging, he underwent multislice cardiac computed tomography (CT) (Lightspeed 16, GE Systems). The gated CT protocol was identical to our protocol used in multislice CT coronary angiography.

Cardiac CT clearly demonstrated the star shaped, Symmetry proximal aortovenous bypass connector (large

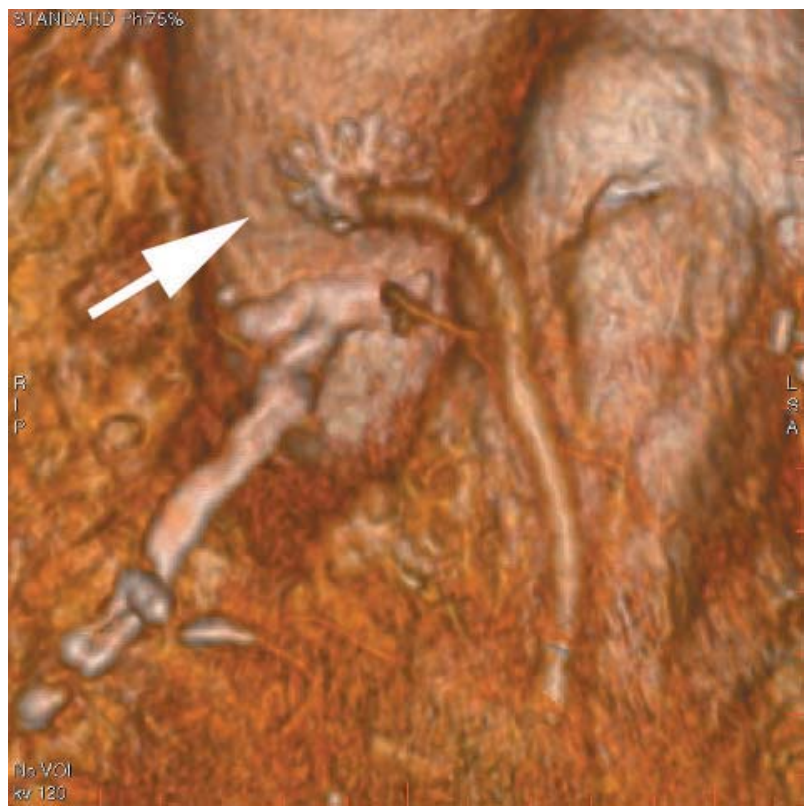

arrow). Patency of the vein graft was evidenced by complete filling of the bypass conduit. Volume rendered (left panel) and curved reformat (right panel) views of the proximal connector and vein graft are shown. There was surprisingly little hardening artefact surrounding the proximal connector. There were areas of discontinuity seen around surgical clips (small arrows) which were consistent with hardening artefact.

This suggests that non-invasive CT angiography may be a viable alternative to evaluating bypass grafts which have anastomoses with proximal connectors.

M Schussler

B L Hamman

JSchussler@heartplace.com

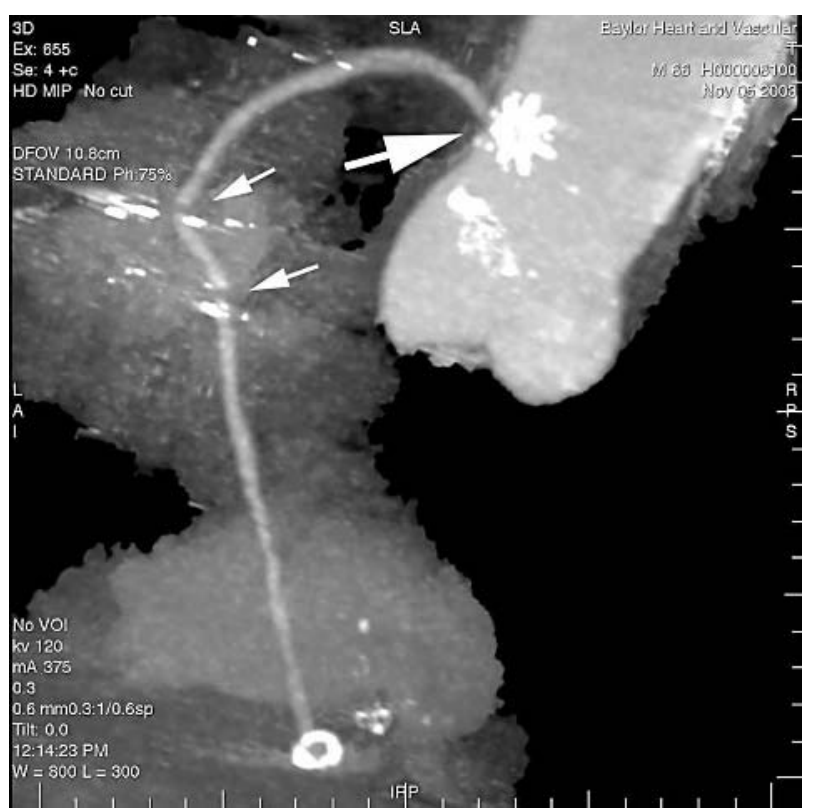

